\title{
The coupled lithium ion diffusion and stress in battery electrodes
}

\author{
Ahmadreza Eshghinejad, Jiangyu Li \\ Mechanical engineering department \\ University of Washington
}

\begin{abstract}
The electrochemical processes in Li-ion batteries are rather complicated, making the insight and guidance from the modeling and simulations essential for the design and optimization of novel electrodes. In this study a continuum model that couples diffusion and mechanics of ion intercalation in electrodes is presented, based on the entropy and mechanical energy associated with the intercalation of ions. The chemical potential difference across the electrode-electrolyte interface is used as the activation energy under the Arrhenius type of kinetics, complemented by governing equation of mechanical equilibrium. The model is implemented into COMSOL finite element platform, and graphite electrodes are used as case studies. It is found that the geometry and topology of the electrodes have important effects on the kinetics as well as capacity of the electrodes, as they have substantial influence on the stress distribution induced by Li-ion intercalation. Higher compressive stress results in lower ion concentration and thus lower capacity, and it also reduces the diffusion rate. As a result, structured electrodes with higher surface area and relaxed stress state tend to have higher capacity and faster rate performance.
\end{abstract}

\section{Introduction}

High performance rechargeable batteries are the critical limiting factor for the next generation portable electronics, electric vehicles, and large scale storage of electricity generated from renewable sources. Although Li-ion batteries have shown the highest energy density and excellent cycling stability among the various types of secondary batteries, the state of art of Li-ion batteries are insufficient to satisfy the ever-increasing demands. While great efforts have been devoted to increasing the energy density of batteries, the 
progress has been slow and the electrodes are considered to be the key bottleneck of the improvements. To overcome such difficulties, there have been attempts to use Li-alloy electrodes such as $\mathrm{Sn}, \mathrm{Si}, \mathrm{Ge}$, and $\mathrm{Al}$, which are shown to provide much higher energy density than conventional Li-ion battery anodes such as graphite. For example, silicon electrodes possess the highest energy density of $4200 \mathrm{mAh} / \mathrm{g}$ [13]. However, very large mechanical deformations are associated with such high capacity, which often causes fracture of the electrode and results in loss of its integrity $[2,21]$.

It is widely known that there is a strong coupling between the electrochemistry and mechanics of Li-ion intercalation in electrodes, which has received a great deal of attention in recent years $[4,19,17,23]$. However most of the studies have focused on relatively simple geometries, making the analytic or semi-analytic solutions possible [17, 23]. Furthermore, while many models have been developed to analyze electrochemical processes in Li-ion batteries, few of such studies have been directed toward designing electrode materials with enhanced performance. We hope to address such issues in this study. In this regard, both thermodynamics and kinetics of electrochemistry are important.

The capacity of electrode is directly related to the amount of ions that can be reversibly inserted and extracted into and out of electrode, and there are numerous continuum based studies addressing ionic transport inside bulk electrode, built on the thermodynamics of intercalation [14]. Due to the strong coupling between electrochemistry and mechanics, recent studies on continuum modeling of ionic diffusion account for the effects of stress as well $[23,22,4,3]$. The thermodynamics of solute atoms in equilibrium inside an elastic host can be traced back to the works of Larch and Cahn [10] and Li et. al [12], and recent studies on the stress-induced diffusion in Li-ion battery electrodes have been based on this general framework. Some researchers have developed more general models of the stress-induced diffusion [5], and Haftbaradaran et. al. have validated diffusion induced by the hydrostatic stress using atomistic simulations [8].

The electrochemistry at the electrolyte-electrode interface is also critical, which is commonly governed by Butler-Volmer relation or its linearized form $[15,7]$. In this kinetics, the activation energy depends on the electrostatic potential difference across the interface, and there is a continuous flux of ions into the electrode until it is filled to its maximum capacity. On the other hand, Bazant et. al. treated reaction rates as governed by Arrhenius kinetics, with the activation energy depending on the chemical potential difference across the interface [18]. Such kinetic model enabled them to predict intercalation phase separation waves propagating through the electrode. 
In this study we adopt a continuum model of diffusion coupled with mechanics as described in section 2. The model considers the effect of nondilute solution that enforces a maximum limit for concentration as the host sites are fully filled. The mechanical energy of ionic defects in the solid electrode are considered as well, with the effect of stress on the activation energy of diffusion accounted for. Finally, the evolution of ionic concentration is derived, with Arrhenius kinetics adopted to address the chemical potential dependent reaction rate at the electrode-electrolyte interface. These governing equations of ion intercalation, mechanics and surface kinetics are implemented and solved in COMSOL Multiphysics in section 4. Finally, the effects of electrode geometry and topology are simulated and discussed.

\section{Continuum modeling framework}

Our continuum modeling framework is based on the following considerations. The diffusion of Li-ions in a solid host is governed by the thermodynamics of intercalation, with the entropy of intercalating non-dilute ions and the mechanical energy required to put an ionic defect into the elastic matrix considered in the chemical potential. The flux of ions and time evolution of ion concentration are derived accordingly, with the interfacial electrochemistry governed by the chemical potential difference across the electrode-electrolyte interface.

\subsection{Ionic transport}

We have adopted interstitial diffusion model for the intercalation of ions into the elastic host electrode wherein the ionic transport is viewed as substitution of ions into the solid host, and the total number of interstitial host in the solid is assumed to be conserved.

We first define the normalized concentration as $c=\frac{\tilde{c}}{c_{\max }}$, where $\tilde{c}$ is the concentration of solute particles in the host and $c_{\max }$ is the corresponding maximum allowable concentration with the unit of $\mathrm{mol} \cdot \mathrm{m}^{-3}$. The ionic transport in the solid host electrode is driven by the difference in its chemical potential, given by

$$
\mu=R T \ln \left(\frac{c}{1-c}\right)-\beta \Omega \sigma_{h} .
$$

In this equation, the first term corresponds to the entropy of solute atoms occupying a fixed set of sites in the host, where $R$ is the universal gas constant and $T$ is the absolute temperature. For dilute solutions $(c \ll 1)$ this term takes the conventional form $R T \ln (c)$. However in non-dilute solutions the entropy approaches zero as the host sites in the solid are fully occupied 
which leads to realization of a maximum concentration. The second term, on the other hand, represents the mechanical potential, which is associated with introducing an inclusion with partial molar volume $\Omega$ under hydrostatic pressure $\sigma_{h}$ into the elastic host. Such mechanical potential was first introduced by Li et al. [12] and Larche and Cahn [10], which is now commonly used in the continuum models of stress-induced diffusion. Note that $\beta$ denotes the extent of coupling between electrochemistry and mechanics, and throughout this study, we only consider fully coupled $\beta=1$ and completely uncoupled $\beta=0$ conditions. Furthermore, we assume that the binding energy (enthalpy) of intercalation is not a function of ions concentration.

At the atomistic scale, diffusion of solute atoms is a series of jumps between vacancies of the host lattice. However the solute atoms need to overcome the energy barrier caused by the interactions with the neighboring atoms. Atomistic simulations reveal the strong dependency of the activation energy of diffusion on internal stress [16], which is also referred as straindependent mobility by Aziz et al. [1]. The energy barrier decreases with a tensile stress acting on the area of diffusion and increases with compressive stress. Hence, the modified diffusivity $D$ as introduced by Haftbaradaran et al. [8] takes the form:

$$
D=D_{o} e^{\beta \alpha \Omega \sigma_{h} / R T},
$$

where $D_{0}$ is the diffusivity in the absence of stress and $\alpha$ is a positive dimensionless coefficient determining the linear dependency of diffusion activation energy on hydrostatic stress.

Finally, the ionic diffusion is governed by the Fick's Law [15], that the flux of species in the host is proportional to the chemical potential gradient and the mobility of species,

$$
\boldsymbol{j}=D_{o} e^{\beta \alpha \Omega \sigma_{h} / R T}\left(-\frac{\nabla c}{1-c}+\beta \frac{\Omega}{R T} c \nabla \sigma_{h}\right) .
$$

Considering balance of ionic species for an infinitesimal volume, we obtain

$$
\frac{\partial c}{\partial t}=-\nabla \cdot \boldsymbol{j}=\nabla \cdot\left[D_{o} e^{\beta \alpha \Omega \sigma_{h} / R T}\left(\frac{\nabla c}{1-c}-\beta \frac{\Omega}{R T} c \nabla \sigma_{h}\right)\right],
$$

which governs the evolution of ion concentration.

\subsection{Interface reaction}

For the electrochemical reaction at the interface between electrode and electrolyte, we adopt what was introduced by Bazant et. al. [18], that the reaction rate is governed by Arrhenius kinetics. The activation energy is 
taken as the chemical potential difference across the electrode-electrolyte interface, which provides the thermodynamic driving force. In contrast, in the standard models of interfacial kinetics, such as Butler-Volmer equation [6], the activation energy only depends on the electrostatic potential difference across the electrode-electrolyte interface. Accordingly, the net flux into the electrode normal to the surface, with unit normal vector $\boldsymbol{n}$, is governed by the difference between insertion and extraction kinetics rates,

$$
\boldsymbol{j}_{s} \cdot \boldsymbol{n}=k_{i n} c_{e} e^{\left(\mu_{e}-\mu_{s}\right) /(R T)}-k_{e x t} c_{s} e^{\left(\mu_{s}-\mu_{e}\right) /(R T)},
$$

where $k_{i n}$ and $k_{\text {ext }}$ are the insertion and extraction reaction rates with the unit of $\mathrm{mol} \cdot \mathrm{m}^{-2} \cdot \mathrm{s}^{-1}$. Concentration and chemical potential of ions at the electrolyte exposed to the solid surface are represented by $c_{e}$ and $\mu_{e}$ respectively, while $c_{s}$ and $\mu_{s}$ are concentration and chemical potential of ions at the solid surface. Note that in the limit of small activation potential $\left(\left|\mu_{e}-\mu_{s}\right| \simeq 0\right)$ and taking $k_{i n}=k_{e x t}=k$, the flux is simplified as $\boldsymbol{j}_{s} \cdot \mathbf{n}=k\left(c_{e}-c_{s}\right)$, which is the linearized form of the Butler-Volmer kinetics that is often used as the active surface ion flux $[7,5]$.

\subsection{Mechanical equilibrium}

As is well known, the diffusion of ions is coupled with molar volume change that induces the internal stress field, which in turn affects diffusion. Thus we need to solve for the stress field within the electrode. In this study the electrode material is assumed to be isotropic, homogeneous and linear elastic. It is commonly assumed that ionic insertion in the electrode generates an eigen-strain that is proportional to the concentration as

$$
\varepsilon_{i j}^{*}=\frac{\Omega}{3} c_{\max } c \delta_{i j},
$$

which generate an internal stress field

$$
\sigma_{i j}=C_{i j k l}\left(\varepsilon_{k l}-\frac{\Omega}{3} c_{\max } c \delta_{k l}\right)
$$

where $C_{i j k l}$ is the stiffness tensor, $\varepsilon_{k l}$ is the total strain and $\delta_{k l}$ is Kronecker delta. Because of the relatively slow electrochemical processes in electrodes, mechanics of intercalation can be solved with quasistatic assumption. The stress field can then be solved from the mechanical equilibrium equation:

$$
\nabla \cdot \sigma=0
$$




\section{Numerical implementation}

In this section numerical implementation of the governing equations in the dimensionless form is explained. We first introduce the normalized coordinate, time and hydrostatic stress,

$$
\hat{\boldsymbol{x}}=\frac{\boldsymbol{x}}{b}, \quad \hat{t}=\frac{t D_{o}}{b^{2}}, \quad \hat{\sigma}_{h}=\frac{\sigma_{h}}{E},
$$

where $E$ is the elastic modulus of the electrode and $b$ is the reference length, taken as the width of periodic unit cell of the electrode (see next sections). In the following formulations $\nabla$ operator acts on a domain with normalized coordinate $\hat{\boldsymbol{x}}$. Hence, the expression for the evolution of ion concentration, found in Equation 4, can be rewritten in the dimensionless form as:

$$
\frac{\partial c}{\partial \hat{t}}=\nabla \cdot\left[e^{\beta \alpha \hat{\sigma}_{h} E \Omega / R T}\left(\frac{\nabla c}{1-c}-\beta \frac{E \Omega}{R T} c \nabla \hat{\sigma}_{h}\right)\right] .
$$

Note that the dimensionless quantity $\frac{E \Omega}{R T}$ determines the coupling strength of ionic diffusion with mechanics.

Assuming that the introduced chemical potential in Equation 1 is valid at the surface of electrode as well, and plugging it in Equation 5, the normal component of the electrode surface flux is found in dimensionless form as:

$$
\hat{\boldsymbol{j}}_{s} \cdot \mathbf{n}=\hat{K}\left(\hat{S} \frac{1-c_{s}}{c_{s}} e^{\frac{\mu_{e}}{R T}+\beta \frac{E \Omega}{R T} \hat{\sigma}_{h}}-\frac{c_{s}^{2}}{1-c_{s}} e^{-\beta \frac{E \Omega}{R T} \hat{\sigma}_{h}-\frac{\mu_{e}}{R T}}\right),
$$

where the dimensionless reaction rate $\hat{K}$ and ratio of insertion to extraction rates $\hat{S}$ are given by

$$
\hat{K}=\frac{k_{e x t} b}{D_{0} c_{\max }}, \quad \hat{S}=\frac{k_{i n s}}{k_{e x t}} .
$$

Finite element method is used to numerically solve the coupled partial differential equations of diffusion and mechanics implemented in COMSOL Multiphysics platform. The evolution equation of concentration is solved via the General PDE mode as,

$$
\frac{\partial c}{\partial \hat{t}}+\nabla \cdot \boldsymbol{\Gamma}=0
$$

where we introduce

$$
\boldsymbol{\Gamma} \equiv e^{\beta \alpha \sigma_{h} E \Omega / R T}\left(-\frac{\nabla c}{1-c}+\beta \frac{E \Omega}{R T} c \nabla \hat{\sigma}_{h}\right) .
$$


Table 1: Material properties of graphite [4, 9, 11] and parameters used in simulations [8].

\begin{tabular}{|c|c|}
\hline Modulus of elasticity $E$ & $15 \mathrm{GPa}$ \\
Poisson's ratio $\nu$ & 0.3 \\
Partial molar volume $\Omega$ & $4.926 \times 10^{-6} \mathrm{~m}^{3} / \mathrm{mol}$ \\
Maximum concentration $c_{\max }$ & $2.64 \times 10^{4} \mathrm{~mol} / \mathrm{m}^{3}$ \\
Chemical potential in electrolyte $\mu_{e}$ & $\mathrm{RT}$ \\
Gas constant R & $8.314 \mathrm{JK}^{-1} \mathrm{~mol}^{-1}$ \\
Temperature T & $300 \mathrm{~K}$ \\
Coefficient $\alpha$ & 0.18 \\
Dimentionless reaction rate $\hat{\mathrm{K}}$ & $3 \times 10^{-2}$ \\
Insertion to extraction ratio $\hat{S}$ & 2 \\
\hline
\end{tabular}

The dimensionless surface flux in Equation 11 is applied as the boundary condition where required. Furthermore, the diffusion-related eigen-strain is treated as thermal strain, and the resulting stress is solved under thermalstress mode in the dimensionless form with plain strain condition. Note that Lagrangian elements are used to discretize the domains.

\section{Discussion and results}

In this section, we study diffusion of Li-ion in electrode, and its coupling with stress, with particular attention paid on the geometry of electrode and the corresponding boundary conditions. In all the simulations, graphite is assumed as the electrode material for demonstration, and its material properties as well as other parameters used in this study are listed in Table 1.

\subsection{Planar electrodes}

We first investigate planar electrodes, as shown in Figure 1. Two configurations are considered, one an infinite electrode extended periodically in lateral direction, while the other with finite width identical to the periodicity of the infinite electrode. Over the top surface, electrode is exposed to electrolyte with constant chemical potential $\mu_{e}=R T$ and concentration $c_{e}=1$ where the electrochemical reaction occurs, and this surface is assumed to be traction free as well. Over the bottom surface, where the electrode is attached to the current collector, no displacement $(u=v=0)$ and normal ionic flux are allowed. For finite electrode, the lateral surface is also exposed to electrolyte with traction free boundary condition. A uniform 


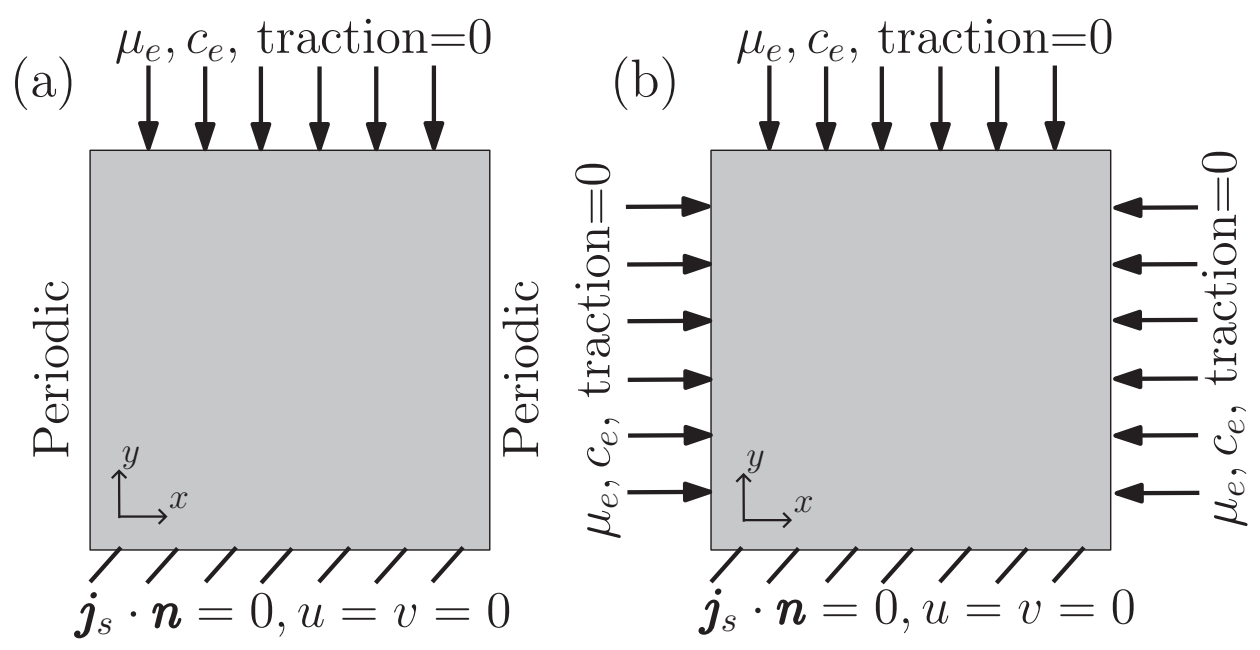

Figure 1: Configuration of planar electrodes; a) the unit cell of an infinite electrode extended periodically in lateral direction; and b) a finite electrode with width identical to that of the periodic unit cell and lateral surface exposed. The reactive surfaces are exposed to an electrolyte with ionic chemical potential of $\mu_{e}$ and concentration $c_{e}$. The bottom surfaces are attached to the current collector without any horizontal and vertical displacements $(u=v=0)$ and normal ionic flux.

initial concentration of $c=0.2$ is assumed, and the simulation stops when the electrode reaches the steady state condition.

In the infinite electrode, due to the periodic and uniform boundary conditions, the concentration profile and stress fields are actually uniform along the lateral direction. Thus, by exploiting the analogy with thermal-stress, the exact stress field at the free surface of the electrode, with ionic concentration $c_{s}$, can be found as:

$$
\sigma_{y}=0 ; \quad \sigma_{x}=\sigma_{z}=-\frac{\nu+1}{3\left(1-\nu^{2}\right)} \Omega E c_{\max } c_{s},
$$

where $\nu$ is the Poisson's ratio and $z$ is out-of-plane direction. This leads to an expression for the chemical potential (Equation 1) of ions at the surface of electrode. Therefore, the analytic expression for the dimensionless normal surface flux from the electrolyte into the infinite electrode, as a function of surface concentration, can be found as

$$
\hat{\boldsymbol{j}}_{s} \cdot \mathbf{n}=\hat{K}\left(\hat{S} \frac{1-c_{s}}{c_{s}} e^{\frac{\mu_{e}}{R T}-\frac{2 \beta E(\nu+1)}{9 R T\left(1-\nu^{2}\right)} \Omega^{2} c_{\max } c_{s}}-\frac{c_{s}^{2}}{1-c_{s}} e^{\frac{2 \beta E(\nu+1)}{9 R T\left(1-\nu^{2}\right)} \Omega^{2} c_{\max } c_{s}-\frac{\mu_{e}}{R T}}\right) .
$$




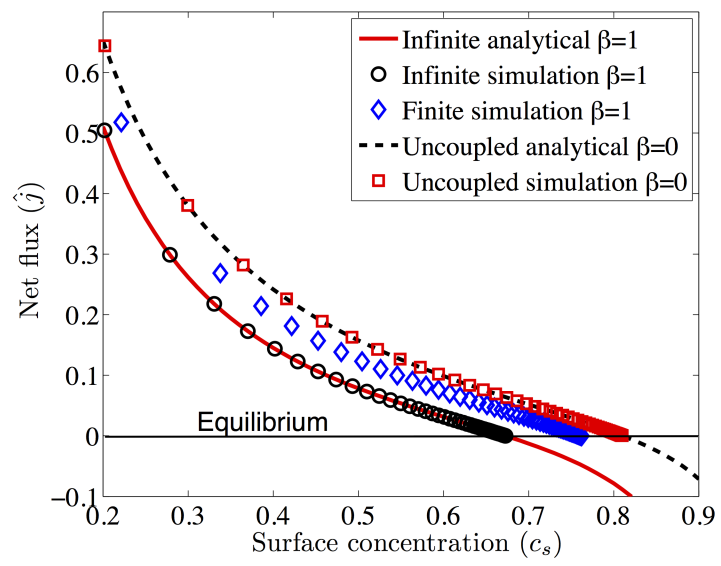

Figure 2: Normal surface flux as a function of surface concentration for the planar infinite and finite electrodes under coupled $(\beta=1)$ and uncoupled $(\beta=0)$ conditions.

The dimensionless normal surface flux as a function of surface concentration $\left(c_{s}\right)$ for the planar infinite and finite electrodes under coupled and uncoupled $(\beta=1,0)$ conditions are shown in Figure 2. The dots in this figure represent time snapshots of the simulations, and the results for the finite electrode are averaged over the exposed surface area. The analytic solutions for the infinite electrode, coupled or uncoupled with stress (Equation 16), are also shown for comparison and validation. Note that the surface flux in the uncoupled model is stress independent and hence is identical for both finite and infinite electrodes. For all cases, the surface flux starts from insertion into the electrode at lower surface concentrations and decreases as the surface concentration increases until reaching the equilibrium state with zero flux. The surface flux is then reversed to extraction for surface concentrations higher than that of equilibrium. This equilibrium concentration (concentration at zero flux) is a function of the stress at the surface of electrode. Therefore the equilibrium concentration is lowest in the infinite electrode, which experiences the highest insertion-induced compressive stress, and is the highest in the uncoupled model with $\beta=0$. The finite electrode's average surface compressive stress and hence its equilibrium concentration lies in between the other two cases (see Figure 4), as the stress state is somewhat relaxed due to the geometry.

At this point, we introduce the capacity of an electrode as the total 


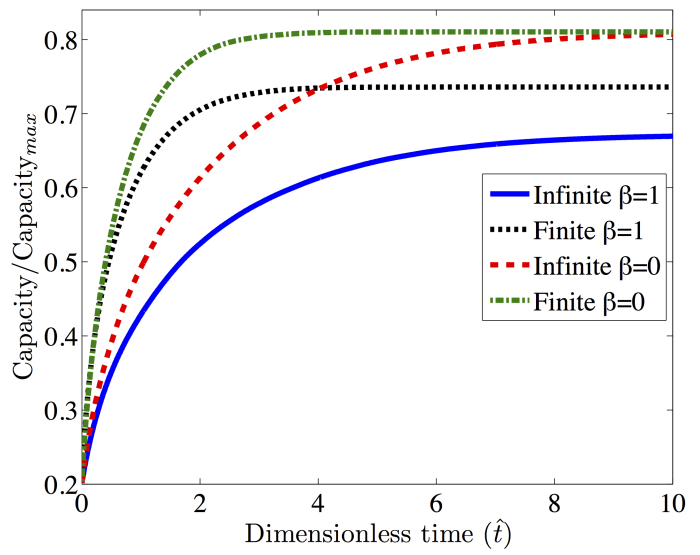

Figure 3: Time evolution of normalized capacity of the planar infinite and finite electrodes for coupled $(\beta=1)$ and uncoupled $(\beta=0)$ models.

amount of ions within that electrode

$$
\text { capacity } \equiv \int_{V} c c_{\max } d V
$$

where $V$ denotes the volume of electrode. The time evolution of normalized capacity $\left(\frac{\text { capacity }}{\text { capacity } \max }\right)$ of the simulated infinite and finite electrodes for coupled and uncoupled models $(\beta=1,0)$ are shown in Figure 3. Note that capacity $_{\max }$ is defined as the capacity of an electrode containing uniform maximum concentration $c_{\max }$. As can be seen, the finite electrode, in both models, reaches the equilibrium state in a shorter time due to its larger surface area exposed to electrolyte. Moreover, while both infinite and finite planar electrodes under the uncoupled model $(\beta=0)$ converge to the same capacity, the coupled model $(\beta=1)$ predicts lower equilibrium capacities due to the stress effect. The equilibrium capacity of the finite electrode is higher compared to the infinite electrode due to the lower level of insertioninduced compressive stress, and such higher capacity in structured carbon electrodes has indeed been reported in the literature [20].

To appreciate the effect of stress on the kinetics as well as the capacity of electrodes, the distributions of equilibrium concentration and hydrostatic stress within the infinite and finite electrodes under the coupled and uncoupled models are shown in Figure 4. The first two rows represent the coupled models and the next two rows represent the uncoupled models. As can be seen, the concentration and hydrostatic stress are uniform within the infinite electrode under both models. This equilibrium concentration is higher 
in the uncoupled model compared to that of the coupled model, also seen in Figure 2. Moreover, within the finite electrode under coupled model (c,d), the location of stress concentration with higher compressive stress exhibits lower equilibrium concentration, illustrating the coupling between mechanics and electrochemistry. Because of this coupling, finite electrode, with lower stress concentration, exhibit higher capacity than the infinite one. It is worth mentioning that in the uncoupled finite electrode $(\mathrm{g}, \mathrm{h})$, while the calculated stress varies, the concentration is uniform and is identical to that of the infinite electrode, because the stress has no influence on the ion concentration.

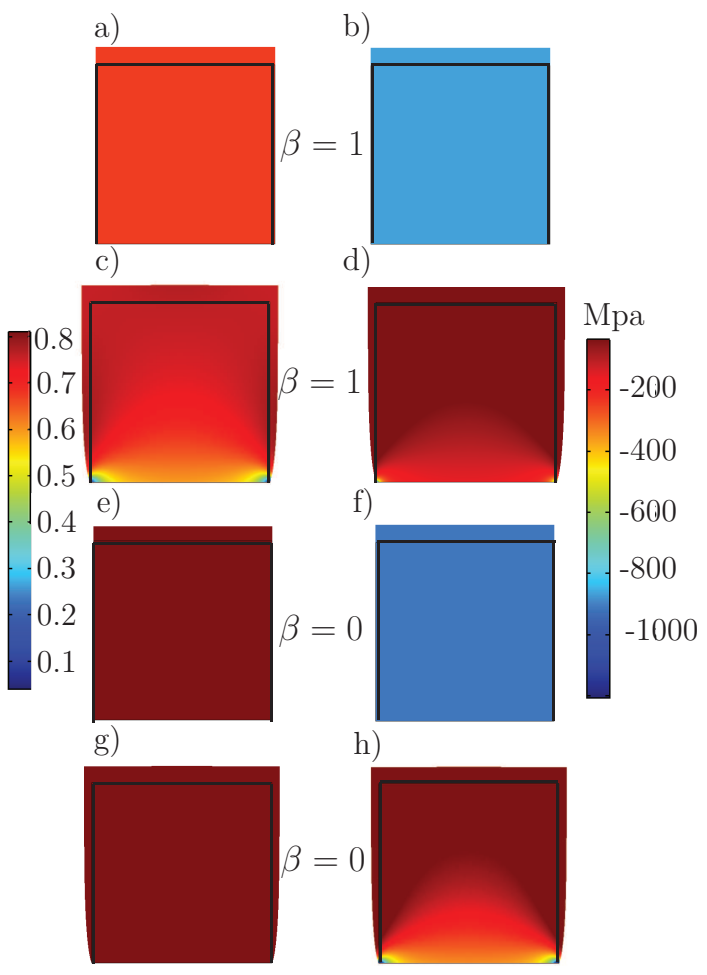

Figure 4: Distribution of equilibrium concentration (left) and hydrostatic stress (right) of planar (a,b,e,f) infinite and (c,d,g,h) finite electrodes under coupled $\beta=1$ and uncoupled $\beta=0$ models.

\subsection{Structured electrodes}

Based on the results in the last subsection, it is clear that geometry of an electrode has substantial influence on the stress distribution induced 


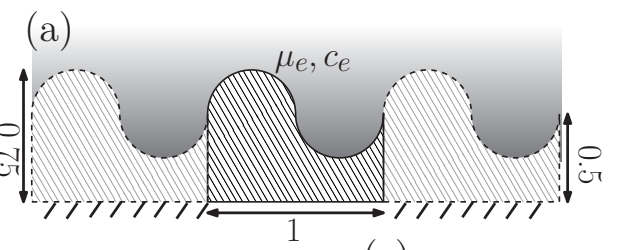

(b)
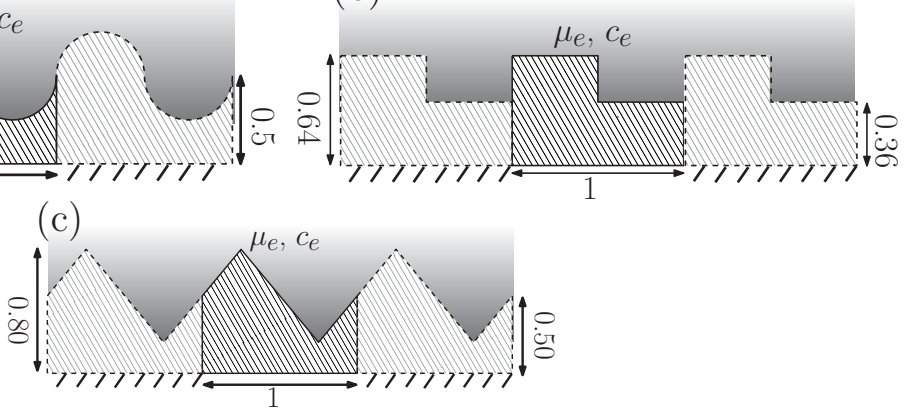

Figure 5: Configurations of structured electrodes; the unit cell of a) semi-circular, b) rectangular and c) triangular electrodes extended periodically in lateral direction. The reactive surfaces are exposed to an electrolyte with ionic chemical potential of $\mu_{e}$ and concentration $c_{e}$. The bottom surfaces are attached to the current collector without any horizontal and vertical displacements $(u=v=0)$ and normal ionic flux.

by the diffusion, which in turn affects the capacity and rate performance of electrodes. In order to further investigate this effect, we study three structured electrodes with periodic semi-circular, rectangular and triangular patterns using the coupled model $\beta=1$, as shown in Figure 5. Note that the period length of the structures is chosen as the reference length scale (see section 3), and the dimensions are set such that the reactive surface area and volume of all the electrodes are kept the same. For comparison, an infinite electrode with the same volume is investigated as well.

In the model configuration depicted in Figure 5, the top surfaces of electrodes are exposed to electrolyte with constant chemical potential $\mu_{e}=R T$ and concentration $c_{e}=1$. The surface reaction over the top surfaces produces a flux normal to the electrode surface as was expressed in Equation 11. The bottom surfaces of the electrodes, attached to the current collector, are considered mechanically fixed with zero normal flux.

The equilibrium concentration along the arc-length of the electrodeelectrolyte interface (top surface) for the structured electrodes are illustrated in Figure 6. The drastic drops of concentration for the triangular and rectangular electrodes corresponds to the concave edges of these electrodes, which experience the highest compressive stress. In contrast, the convex edges accommodate the maximum concentration due to the lowest compressive stress level. The concentration profile over the semi-circular electrode is smooth, yet similar concentration drop can be observed in the concave area. Moreover, the concentration over the pillar section of the rectangular elec- 


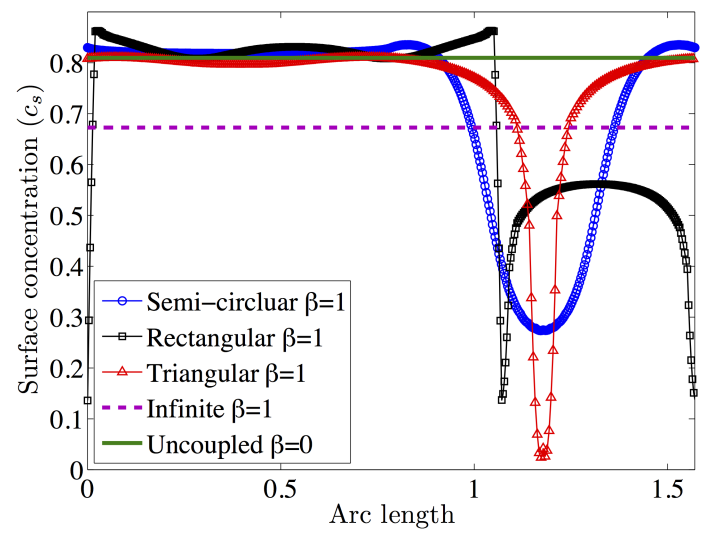

Figure 6: Equilibrium surface concentration along the reactive interface arc length.

trode is comparable to that of the uncoupled model, while the concentration over the base section is lower than that of the infinite electrode due to stress concentration.

The time evolution of normalized capacity of the semi-circular, triangular and rectangular electrodes as well as the infinite electrode are shown in figure 7. Electrodes with different structures converge to different equilibrium capacities, dependent on their respective stress state. The electrode with semi-circular structures converges to the maximum equilibrium capacity due to the the least average compressive stress state, while the electrode with rectangular structures converges to the minimum equilibrium capacity. This low capacity is associated with the high difference between the average hydrostatic stress in the base and pillar sections, which results in high concentration difference between these sections (as was also seen in Figure 6). Note that the time required for the infinite electrode to reach equilibrium state is longer compared to the structured electrodes because of the least exposed area.

The distributions of equilibrium concentration and hydrostatic stress within the structured electrodes are shown in Figure 8. They are uniform within the infinite electrode while spatially varying for the structured electrodes. Because of the coupling, a correlation between concentration and induced hydrostatic stress within the electrodes is observed. For example, the areas close to the concave edges that experience the highest compressive stress accommodate the lowest ion concentration. Moreover, the previously discussed concentration difference between the base and pillar sections of 


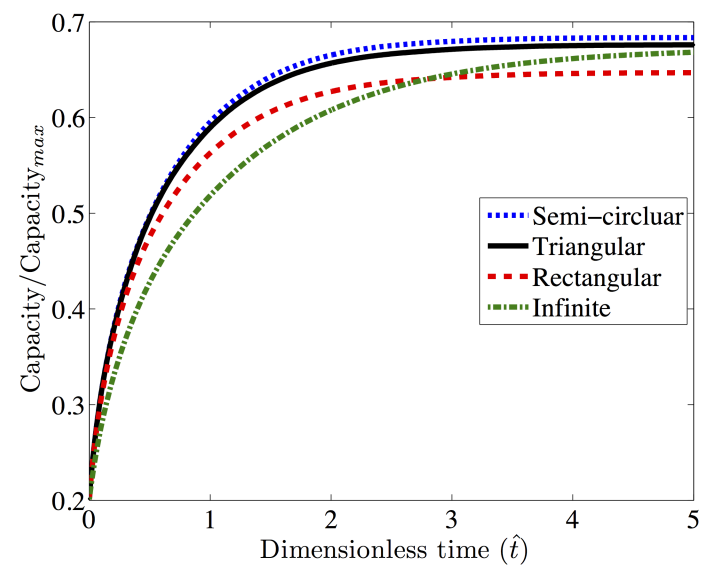

Figure 7: Time evolution of the normalized capacity of the structured electrodes.

the rectangular electrode can be observed as well.

\section{Conclusion}

In this study, a continuum model for the coupled electrochemistry and mechanics in solid host electrodes was developed. The thermodynamics based model accounted for the entropy and mechanical energy associated with intercalation of non-dilute solute ions, resulting in coupled diffusionmechanics relation with the recognition of a maximum concentration of solute ions in the host. Arrhenius type of kinetics was employed at the electrode-electrolyte interface, with chemical potential difference serving as the activation energy, providing an ion flux normal to the electrode surface as a function of ion concentration and hydrostatic stress. The coupled diffusion-mechanics model was implemented into COMSOL multiphysics finite element platform, and graphite electrodes were investigated as case studies.

Planar electrodes with different boundary conditions as well as structured electrodes with different geometries were investigated using the developed model. The electrodes were analyzed in charging condition until reaching an equilibrium with the exposed electrolyte. The coupled model predicted varying equilibrium concentration at the surface depending on the stress state of electrode, illustrating the important role of compressive stress, which tends to reduce the ion concentration, and thus the capacity. Furthermore, faster kinetics is also observed in the structured electrodes due to 


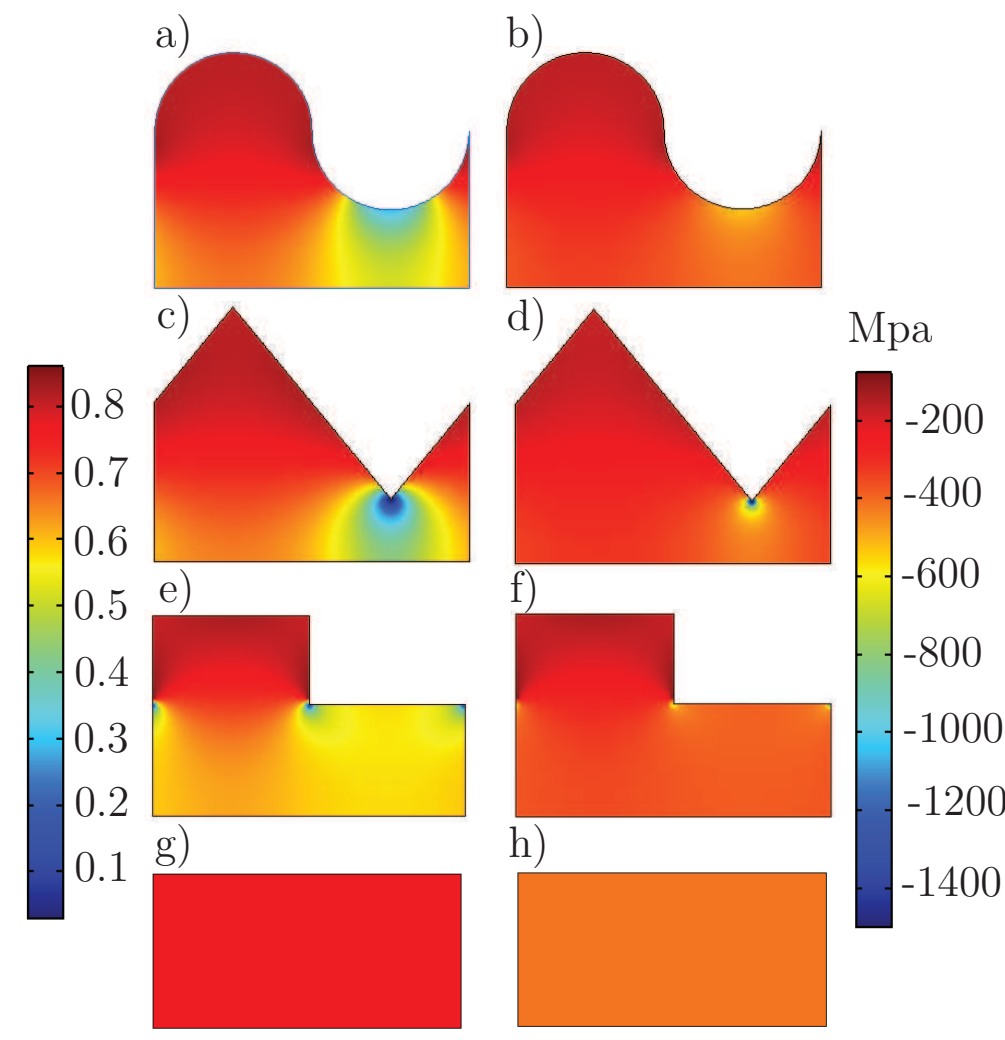

Figure 8: Distribution of equilibrium (a,b,c,g) concentration and (d,e,f,h) hydrostatic stress within a unit cell of the structured electrodes.

larger surface area and reduced stress. Among the geometries considered, semi-circular shaped electrode exhibits the highest capacity, though we believe that it can be further optimized. The presented modeling framework thus can be used to not only understand the effects of stress on the battery performance, but also design and optimize structured battery electrodes with improved performance.

\section{Acknowledgment}

The authors would like to acknowledge University of Washington Clean Energy Institute Graduate Fellowship and NSF (CBET-1435968) for the financial support. 


\section{References}

[1] Michael J Aziz, Paul C Sabin, and Guo-Quan Lu, The activation strain tensor: nonhydrostatic stress effects on crystal-growth kinetics, Physical Review B 44 (1991), no. 18, 9812.

[2] BA Boukamp, GC Lesh, and RA Huggins, All-solid lithium electrodes with mixed-conductor matrix, Journal of the Electrochemical Society 128 (1981), no. 4, 725-729.

[3] Yang-Tse Cheng and Mark W Verbrugge, Evolution of stress within a spherical insertion electrode particle under potentiostatic and galvanostatic operation, Journal of Power Sources 190 (2009), no. 2, 453-460.

[4] John Christensen and John Newman, Stress generation and fracture in lithium insertion materials, Journal of Solid State Electrochemistry 10 (2006), no. 5, 293-319.

[5] Zhiwei Cui, Feng Gao, and Jianmin Qu, A finite deformation stressdependent chemical potential and its applications to lithium ion batteries, Journal of the Mechanics and Physics of Solids 60 (2012), no. 7, $1280-1295$.

[6] Marc Doyle, Thomas F Fuller, and John Newman, Modeling of galvanostatic charge and discharge of the lithium/polymer/insertion cell, Journal of the Electrochemical Society 140 (1993), no. 6, 1526-1533.

[7] R Edwin Garcia, Yet-Ming Chiang, W Craig Carter, Pimpa Limthongkul, and Catherine M Bishop, Microstructural modeling and design of rechargeable lithium-ion batteries, Journal of The Electrochemical Society 152 (2005), no. 1, A255-A263.

[8] Hamed Haftbaradaran, Jun Song, WA Curtin, and Huajian Gao, Continuum and atomistic models of strongly coupled diffusion, stress, and solute concentration, Journal of Power Sources 196 (2011), no. 1, 361370 .

[9] Robert Kostecki and Frank McLarnon, Microprobe study of the effect of li intercalation on the structure of graphite, Journal of power sources 119 (2003), 550-554.

[10] F Larché and JW Cahn, A linear theory of thermochemical equilibrium of solids under stress, Acta Metallurgica 21 (1973), no. 8, 1051-1063. 
[11] James Chen-Min Li, Physical chemistry of some microstructural phenomena, Metallurgical Transactions A 9 (1978), no. 10, 1353-1380.

[12] JCM Li, RA Oriani, and LS Darken, The thermodynamics of stressed solids, Zeitschrift für Physikalische Chemie 49 (1966), no. 3_5, 271-290.

[13] J.W. Park J.A. Ascencio M.H. Kim, S.H. Ahn, Electrochemical characteristics of a si/ge multilayer anode for lithium-ion batteries, Journal of the Korean Physical Society 49 (2006), 1107-1110.

[14] Gholam-Abbas Nazri and Gianfranco Pistoia, Lithium batteries: science and technology, Springer Science \& Business, 2008.

[15] John Newman and Karen E Thomas-Alyea, Electrochemical systems, John Wiley \& Sons, 2012.

[16] David L Olmsted, Rob Phillips, and WA Curtin, Modelling diffusion in crystals under high internal stress gradients, Modelling and Simulation in Materials Science and Engineering 12 (2004), no. 5, 781.

[17] Sindhuja Renganathan, Godfrey Sikha, Shriram Santhanagopalan, and Ralph E White, Theoretical analysis of stresses in a lithium ion cell, Journal of the Electrochemical Society 157 (2010), no. 2, A155-A163.

[18] Gogi K Singh, Gerbrand Ceder, and Martin Z Bazant, Intercalation dynamics in rechargeable battery materials: General theory and phasetransformation waves in lifepo ${ }_{4}$, Electrochimica Acta 53 (2008), no. 26, 7599-7613.

[19] Deyu Wang, Xiaodong Wu, Zhaoxiang Wang, and Liquan Chen, Cracking causing cyclic instability of lifepo $_{4}$ cathode material, Journal of Power Sources 140 (2005), no. 1, 125-128.

[20] Peiqi Wang, Qian Nataly Chen, Shuhong Xie, Xiaoyan Liu, and Jiangyu $\mathrm{Li}$, Enhanced lithium ion storage in nanoimprinted carbon, Applied Physics Letters 107 (2015), no. 4, 043904.

[21] Martin Winter, Jürgen O Besenhard, Michael E Spahr, and Petr Novak, Insertion electrode materials for rechargeable lithium batteries, Advanced materials 10 (1998), no. 10, 725-763.

[22] Xiangchun Zhang, Ann Marie Sastry, and Wei Shyy, Intercalationinduced stress and heat generation within single lithium-ion battery cathode particles, Journal of The Electrochemical Society 155 (2008), no. 7, A542-A552. 
[23] Xiangchun Zhang, Wei Shyy, and Ann Marie Sastry, Numerical simulation of intercalation-induced stress in li-ion battery electrode particles, Journal of the Electrochemical Society 154 (2007), no. 10, A910-A916. 